\title{
Moyamoya disease in pediatric patients: outcomes of neurosurgical interventions
}

\author{
Anand Veeravagu, B.S., Raphael Guzman, M.D., Chirag G. Patil, M.D., \\ Lewis C. Hou, M.D., Marco Lee, M.D., Ph.D., and Gary K. Steinberg, M.D., Ph.D. \\ Department of Neurosurgery, Stanford University School of Medicine, Stanford, California
}

\begin{abstract}
$\checkmark$ Neurosurgical interventions for moyamoya disease (MMD) in pediatric patients include direct, indirect, and combined revascularization procedures. Each technique has shown efficacy in the treatment of pediatric MMD; however, no single study has demonstrated the superiority of one technique over another. In this review, the authors explore the various studies focused on the use of these techniques for MMD in the pediatric population. They summarize the results of each study to clearly depict the clinical outcomes achieved at each institution that had utilized direct, indirect, or combined techniques. In certain studies, multiple techniques were used, and the clinical or radiological outcomes were compared accordingly.

Direct techniques have been shown to aid a reduction in perioperative strokes and provide immediate revascularization to ischemic areas; however, these procedures are technically challenging, and not all pediatric patients are appropriate candidates. Indirect techniques have also shown efficacy in the pediatric population but may require a longer period for revascularization to occur and perfusion deficits to be reversed. The authors concluded that the clinical efficacy of one technique over another is still unclear, as most studies have had small populations and the same outcome measures have not been applied. Authors who compared direct and indirect techniques noted approximately equal clinical outcomes with differences in radiological findings. Additional, larger studies are needed to determine the advantages and disadvantages of the different techniques for the pediatric age group.
\end{abstract}

(DOI: 10.3171/FOC/2008/24/2/E16)

\section{KEY WORDS • direct bypass - moyamoya disease - superficial temporal artery • middle cerebral artery anastomosis}

$\mathrm{M}$ OYAMOYA disease was first described in the Japanese medical literature in 1957 by Takeuchi and Shimuzu. ${ }^{50}$ The term "moyamoya" (Japanese for "puff of smoke") was coined by Suzuki and Takaku ${ }^{48}$ in 1969 to describe the diagnostic appearance of angiogenesis on a catheter angiogram. Since its initial discovery, the features of the disease have become clearer; however, its origin remains unknown. Moyamoya disease is a chronic cerebrovascular disease characterized by stenosis or occlusion of the bilateral terminal internal carotid arteries, resulting in the characteristic development of an abnormal vascular network in the areas of the arterial occlusions..$^{3,54} \mathrm{It}$ has been hypothesized that in the setting of arterial stenosis or occlusion, hypoxic regions of the brain induce deep col-

Abbreviations used in this paper: $\mathrm{ACA}=$ anterior cerebral artery; EDAMS = encephaloduroarteriomyosynangiosis; EDAS = encephaloduroarteriosynangiosis; EGPS = encephalogaleo(periosteal)synangiosis; EMS = encephalomyosynangiosis; $\mathrm{MCA}=$ middle cerebral artery; MMA = middle meningeal artery; $\mathrm{MMD}=$ moyamoya disease; PCA = posterior cerebral artery; STA = superficial temporal artery; TIA $=$ transient ischemic attack. lateral flow through dilated and tortuous perforating arteries. This revascularization strategy is orchestrated by the expression of various angiogenic signaling cascades. ${ }^{29,42}$

Although recent literature shows a rising number of MMD diagnoses in non-Asian populations, the exact incidence has not been determined. ${ }^{6}$ Among the Asian population the disease often affects more children than adults, with each age group presenting with a unique clinical syndrome. Whereas some authors have reported similar presentation characteristics in North American patients, ${ }^{45}$ others have noted distinct differences from Asian populations. ${ }^{1,257}$ In children, the most common presentation is cerebral ischemia. In the Scott and colleagues' ${ }^{45}$ study of 143 pediatric patients with MMD in North America, nearly all of the patients presented with symptoms of either stroke or TIA; similar findings were revealed in European studies. ${ }^{18,40}$ Moreover, in large populations of Asian patients, $\sim 40 \%$ of those younger than 10 years of age presented with a TIA and nearly $30 \%$ presented with cerebral infarction ${ }^{23}$ some presented with headaches and seizures. Note, however, that adults most commonly present with cerebral hemorrhage. In the Kim et al. ${ }^{22}$ study, $66 \%$ of 
patients with adult-onset MMD presented with hemorrhage. ${ }^{60}$ The annual risk of bleeding in adult patients with untreated MMD has been suggested to be $>7 \%$ per year. ${ }^{26}$

The diagnostic guidelines for identifying patients with MMD differ in the various centers around the world. The Research Committee on MMD of the Ministry of Health and Welfare in Japan has identified 4 criteria necessary for the diagnosis of MMD: 1) stenosis or occlusion of the terminal portion of the internal carotid artery; 2) a coexisting abnormal vascular network in the base of the brain or basal ganglia; 3) bilateral vascular involvement; and 4) no other identifiable cause of existing symptoms. ${ }^{5}$ These guidelines have been modified on a case-by-case basis at various institutions around the US. Although classically a bilateral disease, MMD can occur unilaterally. In our series, $75 \%$ of patients with equivocal or mild stenotic changes in the initially unaffected side eventually progressed to bilateral MMD at a mean follow-up time of 12.7 months. ${ }^{17}$

Moyamoya disease is diagnosed and staged by using catheter angiography. The appearance and extent of stenosis, occlusion, and revascularization is divided into 6 distinct stages: ${ }^{48}$ Stage 1 , narrowing of the internal carotid arteries; Stage 2, initial appearance of moyamoya vessels; Stage 3, further definition of collateral vessels; Stage 4, minimization of collateral vessels; Stage 5, reduction of collateral vessels; and Stage 6, disappearance of collateral vessels. These stages have been used to stratify surgical candidates and monitor treatment efficacy.

With a morbidity rate of $>70 \%$ in untreated patients, ${ }^{37,58}$ surgical intervention has become the standard therapy in patients with MMD. ${ }^{22,43,44}$ Surgical interventions have been divided into direct and indirect bypass techniques. The direct bypass techniques proposed thus far include STAMCA, occipital artery-MCA, and MMA-MCA anastomoses. The indirect techniques consist of EMS, EDAS, EDAMS, encephalomyoarteriosynangiosis, multiple cranial bur holes, and omental transposition. Other options such as cervical carotid sympathectomy and superior cervical perivascular ganglionectomy have also been proposed.

For this report, we performed a PubMed-based literature search focusing on studies in which authors quantified outcomes of direct, indirect, and combined (direct + indirect) bypass techniques for MMD in pediatric patients. We summarized published data to provide insight into the current standard of care for children with MMD.

\section{Review of Current Neurosurgical Interventions}

Neurosurgical techniques for the treatment of MMD have been grouped into 2 main categories: direct anastomotic and indirect nonanastomotic. The principal difference between the 2 strategies lies in the method of cerebral reperfusion. Whereas direct methods attempt to harness nascent vasculature with new supply arteries to perfuse affected areas of the brain, indirect methods aim to stimulate the development of a new vascular network over time.

Currently there are no clear data to support either direct or indirect revascularization techniques in the pediatric population with MMD. The method depends on the age of the patient, extent of disease, and anatomic size of the vasculature, a direct or indirect technique will be chosen. The decision is largely based on the surgeon's comfort in per- forming a particular procedure. Indirect vascularization leads to delayed collateralization, whereas direct bypass can immediately perfuse ischemic areas. Some authors have asserted that indirect techniques do not lead to a predictable vascularization pattern and therefore may not resolve the associated moyamoya vascular formation and may also carry an increased risk for postoperative stroke., ${ }^{4,55}$ Thus, others have suggested combining the direct and indirect techniques to take advantage of immediate revascularization with the security of more diffuse neovascularization. This approach has now evolved to STA-MCA direct bypass combined with EDAS, EDAMS, or EMS.

\section{Direct Bypass Techniques}

The STA-MCA bypass technique was first performed in 1967 by Donaghy and Yaşargil ${ }^{56}$ and was used in 1973 by Kikuchi and Karasawa ${ }^{19}$ to treat MMD. ${ }^{39}$ The direct technique has been well documented in the medical literature. ${ }^{60}$ At our institution, direct revascularization techniques are preferred and have been performed whenever anatomical and disease characteristics allow it. Preoperative angiography must include selective external carotid artery injections, and an STA branch must be visualized. During anesthesia normocapnia is maintained, blood pressure is supported in the normal to high range, and the patient is cooled to $33^{\circ} \mathrm{C}$ by using either the Celsius Control System (INNERCOOL Therapies) or surface cooling. Pediatric patients are kept in a state of mild hypothermia by using surface cooling. Given the propensity for intraoperative stroke, the anesthetic requirements of each patient must be carefully considered. ${ }^{46}$ The parietal, or less often the frontal, branch of the STA (donor vessels) is located using Doppler ultrasonography and is dissected along its path. Great care is taken to avoid excessive manipulation of the donor arteries, and papaverine is applied to minimize spasm and maintain patency. The donor vessel is kept intact until the bypass can be started. At this point, in children older than 4 years of age an intraoperative decision is made as to whether a direct or indirect technique should be used. The minimum diameter of the STA and MCA is $0.7-0.8 \mathrm{~mm}$ at our institution. The temporalis muscle is then dissected, and a bone flap is centered along the sylvian fissure. The dura mater is carefully opened, and the cortex is surveyed for the largest artery. Ideally, an $\mathrm{M}_{4}$ branch $\geq 1 \mathrm{~mm}$ in diameter and free of microperforating vessels will be found. Once the recipient vessel has been identified, the arachnoid is opened and the recipient artery is dissected free. A temporary atraumatic microvascular clip is placed across the proximal STA. A fish mouth cut is used at the distal STA at an angle to create an opening diameter greater than that of the recipient vessel. The patient is given a bolus dose of thiopental for neuroprotection (usually inducing electroencephalography burst suppression), atraumatic microvascular clips are applied to both ends of the recipient vessel, and an arteriotomy is performed. A 10-0 monofilament suture is used to anchor both ends of the donor and recipient vessels. Multiple single stitches are continued to secure the 2 vessels in place, ensuring that the back wall of the vessel has not been captured within the suture. The distal MCA clip and then the proximal MCA clip are removed. Finally, the STA clip is removed and bidirectional flow through the MCA is assessed via Doppler ultra- 


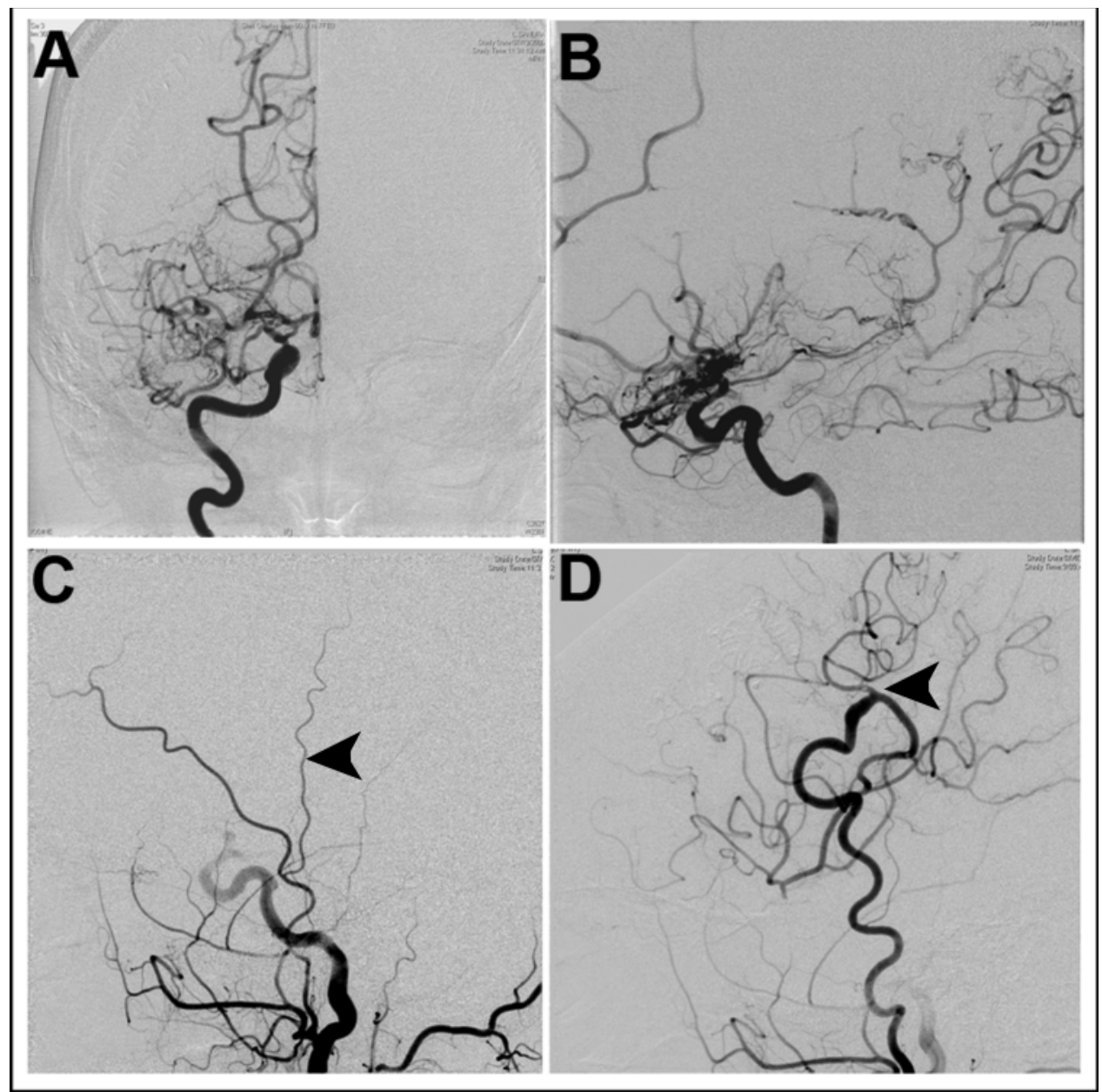

FIG. 1. Angiograms obtained prior to bypass surgery, anteroposterior (A) and lateral (B) views, showing characteristic MMD vascularization. External carotid artery injection angiogram (C) showing the STA (arrow). Note the small size of the vessel. Angiogram (D) obtained 6 months after anastomosis, revealing a robust STA-MCA bypass (arrow) with significant enlargement of the STA.

sonography. All flows are quantified using a probe (Transonic Systems, Inc.; Fig. 1).

Although less studied in the context of MMD, vein and radial artery grafts have been used. Ishii and colleagues ${ }^{10}$ have completed STA-MCA and STA-ACA anastomoses using cephalic vein grafts. The literature describing clinical outcomes after using vein grafts for MMD is scarce. ${ }^{30,31}$

\section{Indirect Bypass Techniques}

Encephalomyosynangiosis was first applied to MMD by Karasawa and associates ${ }^{15}$ in the late 1970s and has now been widely used as an initial surgical intervention. ${ }^{9,27,51,53,59}$ This technique involves implanting the temporalis muscle on the lateral brain surface and securing it to the dural edges. The temporalis muscle is an ideal candidate due to its anatomical location, rich blood supply, and potential for dense vascular collateralization. The goal is for the temporalis muscle to form a bridging arterial supply that will aid in revascularizing the ischemic area of the brain. Disadvantages of this technique include possible mass effect, increased seizures, and cosmetic sacrifice.

Encephaloduroarteriosynangiosis was first developed by
Matsushima and colleagues ${ }^{34}$ in 1979 and is our preferred technique for indirect revascularization in MMD. This technique requires exposure and dissection of the parietal portion of the STA with preservation of vascular flow. After splitting the temporalis muscle, a craniotomy over the sylvian fissure is performed. The dura is carefully opened and multiple arachnoid openings are created. The exposed portion of the vessel is then laid onto the cortical surface, and the cuff surrounding the vessel is sutured to the dural edges (Fig. 2). 12,45,52

Based on the aforementioned techniques, another procedure that combines EDAS and EMS was created. Encephaloduroarteriomyosynangiosis ${ }^{24}$ was developed with the hypothesis that increased neovascularization from the temporalis muscle and STA would be more likely to fully perfuse the affected area. This procedure also requires the creation of dural flaps that are folded into the subdural/epiarachnoid space to allow MMA participation in angiogenesis.

Similarly, another technique that makes use of the dural vascular supply has been termed "EGPS." In this procedure an epigaleal scalp dissection is performed, and a galeoperiosteal flap is incised in an $\mathrm{H}$ pattern. A midline 

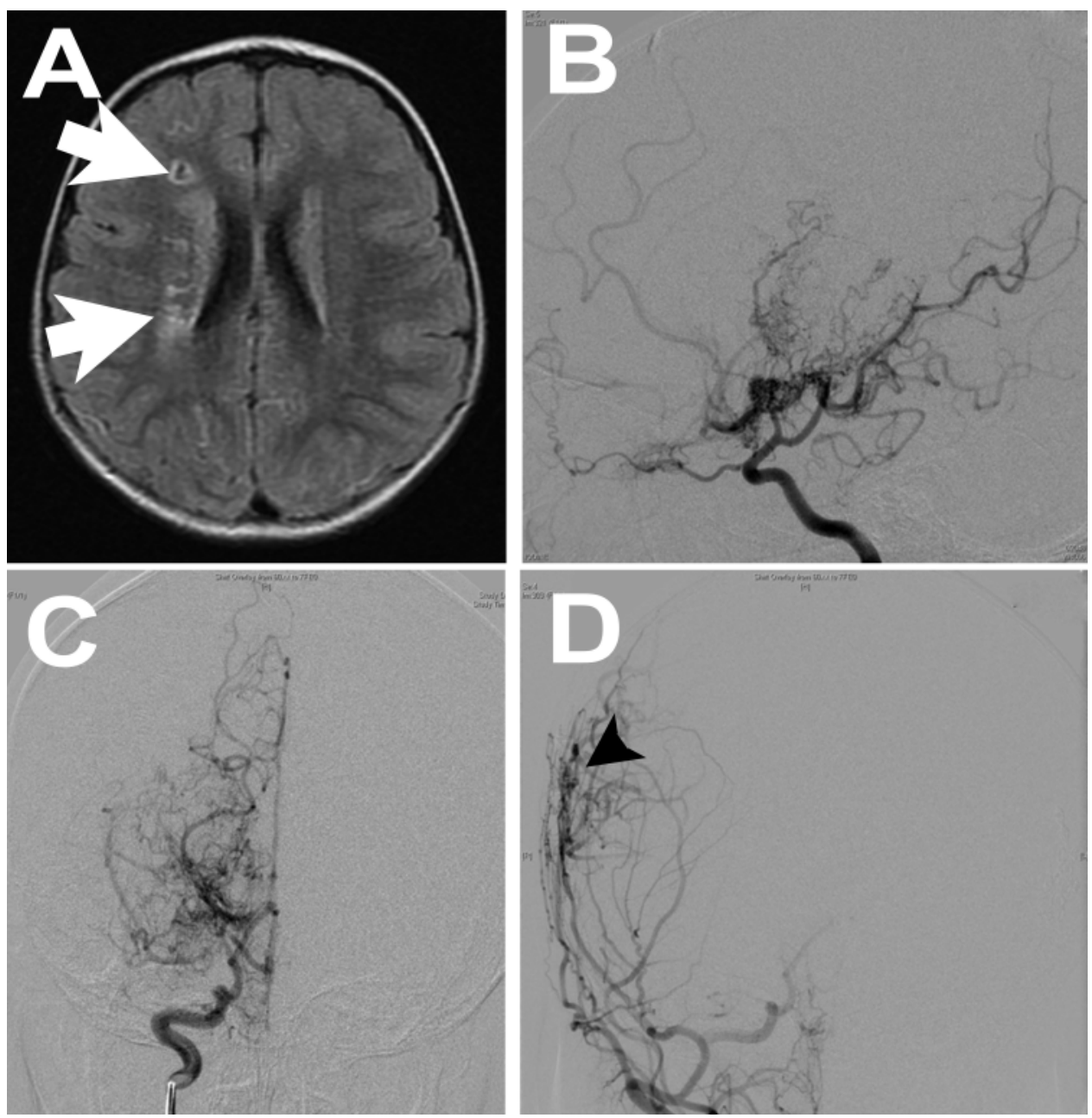

FIG. 2. Axial FLAIR magnetic resonance image (A) revealing evidence of previous right-sided strokes (arrows). Preoperative right-sided carotid artery injection angiograms, lateral (B) and anteroposterior (C) views, demonstrating severe MMD with high-grade stenosis of the terminal carotid artery and occlusion of the MCA as well as formation of moyamoya vessels. Postoperative angiogram (D) obtained after EDAS, showing indirect neovascularization (arrow).

bifrontal craniotomy crossing the superior sagittal sinus is performed. The dura mater is incised separately in both hemispheres, and the arachnoid membrane is dissected. The previously created dural flaps are inserted into each interhemispheric fissure, and the galeoperiosteal flap is sutured to the margin of the dura mater. This technique is well documented and illustrated by Park et al. ${ }^{38}$ and Kim et al..$^{23}$

The transfer of omental tissue to the surface of the cortex also has been attempted. ${ }^{47}$ Karasawa and associates ${ }^{16}$ dissected omental tissue with the gastroepiploic artery and vein for transplantation to the MCA and ACA territories of the brain. These authors concluded that this procedure may be appropriate for those with MMD disease in the area of ACA or PCA; however, it requires harvesting omental tissue via laparotomy and a large craniotomy that will support the omental tissue and thus may carry an increased surgical risk.

Another technique that is technically less challenging is multiple cranial bur holes for cerebral revascularization. ${ }^{40}$ This technique was developed after vasculogenesis was observed in patients with ventriculostomies requiring cranial bur holes. Thus, strategically placed bur holes in areas of cerebral hypoperfusion might aid in stimulating vascular formation between underlying cortex and dura mater, effectively providing an arterial supply to hypoxic areas of the brain.

\section{Outcomes of Direct, Indirect, and Combined Bypass Techniques}

The direct technique is generally accepted as providing immediate vascularization to the MCA territory affected by progressing disease; however, the progression of MMD beyond the territory supplied by the MCA (e.g., the ACA), is not always corrected by the traditional STA-MCA bypass. Therefore, some centers have developed indirect techniques and have attempted to combine direct and indirect techniques to reduce the possibility of disease progression beyond the therapeutic capability of the STA-MCA bypass. Here we discuss the outcomes of various studies in which the authors applied the direct bypass technique, a combination of the direct plus indirect techniques, or an indirect technique alone for MMD in pediatric patients (Table 1). 
TABLE 1

Literature review of studies on outcomes of neurosurgical interventions for moyamoya disease in children*

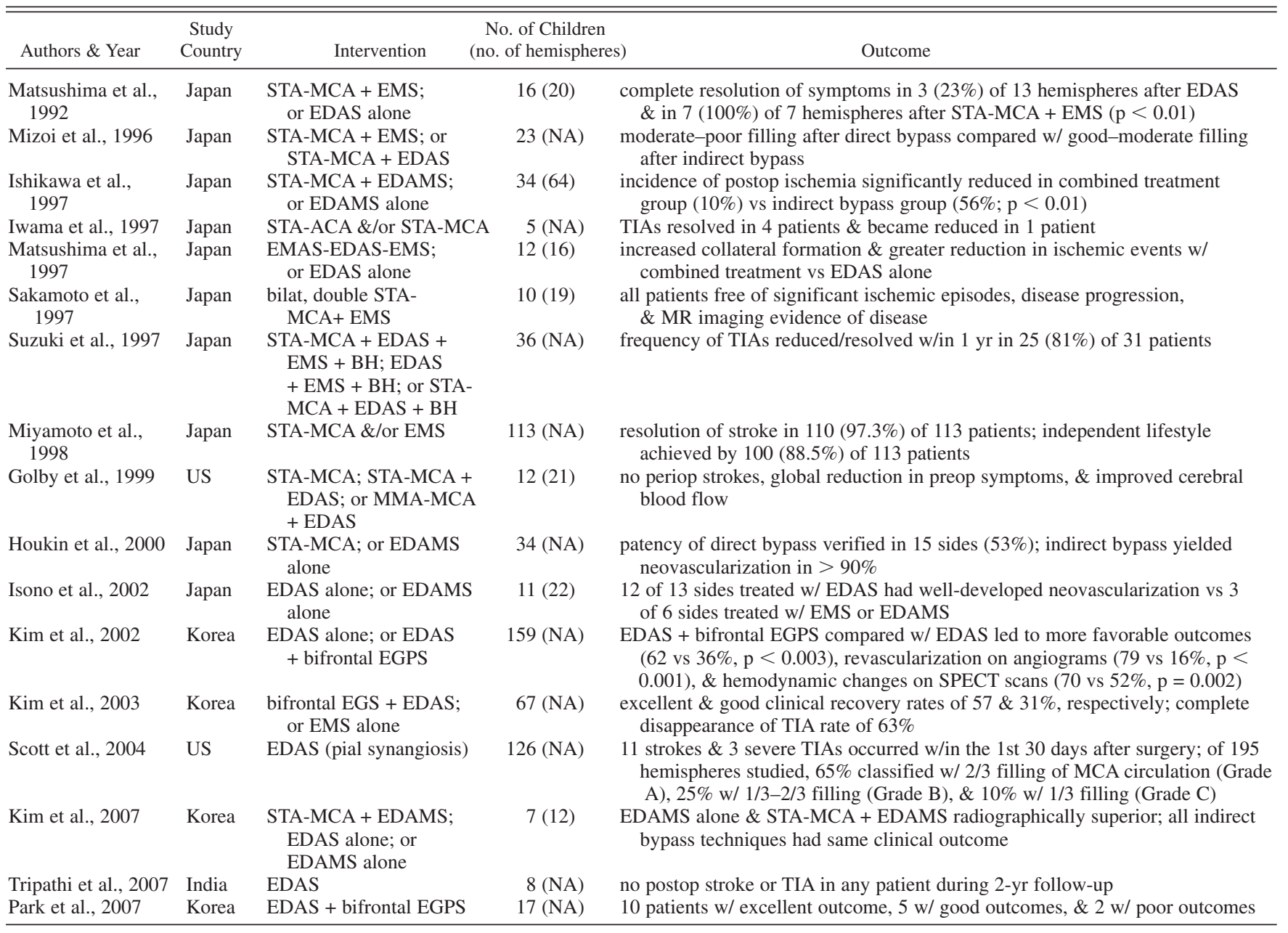

$* \mathrm{BH}=$ bur hole; EMAS $=$ encephalomyoarteriosynangiosis; $\mathrm{MR}=$ magnetic resonance; $\mathrm{NA}=$ not available; SPECT $=$ single-photon emission computed tomography.

The independent use of a direct STA-MCA bypass in pediatric patients with MMD rarely has been reported in the literature. Previously, we described the outcomes of direct STA-MCA bypass and STA-MCA + EDAS in a pediatric population. ${ }^{4}$ In a group of 12 patients, 21 hemispheres were treated with direct bypass techniques. Concomitant EDAS was applied in 6 hemispheres by using a second branch of the STA, 1 patient underwent MMAMCA anastomosis plus EDAS, and 1 patient underwent omental transposition. Outcomes in this population included a global reduction in preoperative symptoms and no perioperative strokes. Some patients experienced transient perioperative neurological symptoms that the authors attributed to possible impaired autoregulation of the cerebral vasculature. Cerebral blood flow analysis in many of these patients revealed increased flow in 68 of the 76 regions examined, indicating the maintenance of patent anastomotic junctions. It was also shown that cerebral blood flow and response to acetazolamide challenge were significantly improved in both MCA and PCA territories.
Direct STA-MCA bypass has also been coupled with and compared to EMS in a study by Mizoi et al..$^{36}$ In their group of 23 patients, 7 were younger than 16 years and underwent combined bilateral direct and indirect procedures. The authors quantified the clinical and radiographic outcomes of each procedure as either high/good, medium/moderate, or low/poor based on distinct criteria for direct filling versus indirect collateralization. ${ }^{32}$ Of the 13 hemispheres in pediatric patients who had undergone direct bypass, medium filling occurred in 7 sides and low filling in 6; none of the hemispheres had high filling. The indirect pediatric group consisted of 14 sides and showed better angiographic results. Collateralization was good in 9 sides and moderate in 5. The authors showed that the effectiveness of the indirect procedure declined as patient age increased. In this particular study, most patients underwent the STA-MCA-EMS procedure bilaterally, which prohibited clinical outcome comparisons. All but 3 patients experienced a resolution in their presenting symptoms, whether ischemic or hemorrhagic. 
Sakamoto and associates ${ }^{42}$ altered the direct bypass technique by including a double STA-MCA anastomoses and EMS. Specifically, both the frontal and parietal branches of the STA were harvested as donor vessels for the recipient MCA. The study included 20 pediatric hemispheres, and the combined procedure was undertaken in 19. Results from the study showed that of the 38 completed anastomoses, 37 maintained patency on angiography at the 1- to 2-month follow-up. Clinical outcome mirrored radiographically demonstrated success; none of the 10 patients showed any significant ischemic episodes, disease progression, or the development of mental retardation at a mean follow-up of 4 years (range 1-10 years). The authors concluded that because the diameter of the STA in younger patients is not large enough to perfuse the deprived area immediately after a single STA-MCA bypass, ${ }^{13}$ double anastomoses combined with EMS may be appropriate for children younger than 10 years of age.

The direct bypass technique has also been augmented with EDAMS by Ishikawa et al. ${ }^{11}$ In their study, 34 pediatric patients underwent surgical treatment in 64 sides for MMD. They performed STA-MCA + EDAMS in 48 sides (combined group) and indirect bypass surgery alone in 16 sides (indirect group). They divided outcomes into perioperative and postoperative events. Perioperative ischemic events occurred in 5 indirect surgeries $(31 \%)$ and in 6 combined surgeries $(13 \%)$, but the difference between the 2 groups was not significant. Postoperatively, the incidence of ischemic events was significantly reduced in the combined group (10\%) compared with that in the indirect group $(56 \% ; \mathrm{p}<0.01)$. The authors also showed the long-term outcome to be unrelated to the type of surgical intervention applied. They assert that even in the setting of reduced ischemic events, the direct bypass technique was not more efficacious than the indirect technique in preventing the deterioration of intelligence as measured using the Wechsler Intelligence Scale for Children-Revised.

In a similar more recent study, Kim et al..$^{21}$ used a combined technique to determine if it was more efficacious than an indirect procedure alone. In that study, STA-MCA + EDAMS was completed in 12 sides in 7 children and was compared with EDAS or EDAMS alone. The goal was to determine if patients treated with the aforementioned methods showed significant differences in the postoperative radiographic or clinical outcome. Although the authors found that patients who had undergone EDAMS or STAMCA + EDAMS were more likely to demonstrate revascularization on angiography, the clinical outcomes of the 3 procedures were not significantly different, which is consistent with data reported by Ishikawa et al. ${ }^{11}$

Encephaloduroarteriomyosynangiosis procedures have also been directly compared with STA-MCA bypass. In a study by Houkin et al. ${ }^{8} 34$ children underwent either EDAMS or direct STA-MCA bypass. The authors found that patency of the direct bypass was much more difficult to achieve, with only 15 sides (53\%) verified on postoperative digital subtraction angiography. The indirect procedure showed neovascularization in $>90 \%$ (MMA or deep temporal artery) of hemispheres. Although clinical outcomes were not discussed in that study, the same group concluded in another article that neither direct nor indirect methods were significantly better at preventing long-term ischemic attack. However, direct techniques were far better in preventing perioperative ischemic complications, possibly due to the newly enabled immediate revascularization. ${ }^{7}$

The EDAS procedure has also been compared with STAMCA + EMS. In a study by Matsushima et al. ${ }^{32}$ in 16 children, 13 hemispheres were treated with EDAS and 7 with STA-MCA + EMS. Complete resolution of symptoms was associated with $3(23 \%)$ of 13 EDAS-treated hemispheres and in $7(100 \%)$ of 7 STA-MCA + EMS-treated hemispheres $(\mathrm{p}<0.01)$. The STA-MCA anastomosis together with EMS was superior to EDAS alone in both the development of collateral circulation $(p<0.05)$ and postoperative clinical improvement $(p<0.01)$. At the author's institution, EDAS led to a 20-30\% rate of failed collateral vascularization, which prompted the authors to begin using the STA-MCA + EMS procedure. They hypothesized that the narrow contact site of EDAS may predispose the ischemic area of the brain to incomplete collateralization. They concluded that EDAS is appropriate as a second-line therapy for MMD in pediatric patients, whereas STA-MCA + EMS remains the most efficacious and should be considered first-line therapy.

Certain indirect procedures have been studied in isolation. In a study by Scott et al., ${ }^{45}$ representing one of the largest single-institution series of pediatric patients with MMD, 126 children underwent pial synangiosis. In that study, the authors reviewed the outcomes of this procedure over the 17 years that it was performed. Of the 126 patients who had undergone surgery, 11 suffered from postoperative stroke and 3 from severe TIA. Angiographic outcome revealed that $65 \%$ of 195 hemispheres showed filling of $2 / 3$ of the MCA circulation; 25\%, 1/3-2/3 filling; and 10\%, $1 / 3$ filling. Furthermore, the synangiosis-induced collateral vessels remained functional and intact for as long as 9 years postoperatively. The authors concluded that $75 \%$ of children who underwent this procedure continue to lead independent and normal lives. Similar EDAS procedures have been shown to yield comparable efficacy in pediatric patients in other parts of the world.52

Encephaloduroarteriosynangiosis has also been compared with other indirect techniques such as EMS and EDAMS. Isono and colleagues ${ }^{12}$ found that in their study of 22 pediatric hemispheres, the EDAS resulted in greater revascularization than the EMS or EDAMS procedure (92 vs $50 \%$, respectively). As the authors mention, however, the treatment arms may not have consisted of patients with equal preoperative cerebral ischemia. Furthermore, none of the 11 patients experienced postoperative stroke, which may highlight the distinction between angiographic and clinical outcomes.

In a study by Kim et al., ${ }^{23}$ the surgical results of EDAS was compared with those of EDAS + bifrontal EGPS. In their study of 159 pediatric patients, 67 underwent EDAS and 92 underwent EDAS + bifrontal EGPS. Data showed that EDAS + bifrontal EGPS significantly improved ACA symptoms ( 81 vs $40 \%, \mathrm{p}<0.015)$, revascularization on angiograms (79 vs $16 \%, \mathrm{p}<0.001$ ), and hemodynamic changes on single-photon emission computed tomography scans (70 vs $52 \%, \mathrm{p}<0.002)$. Despite these differences in radiographic outcomes, however, the incidence of postoperative infarction was not significantly different, and thus final clinical outcomes were not related to the surgical modality chosen. This study again demonstrates that even though radiographic outcomes can differ significantly, clin- 
ical outcomes can remain equal. In a smaller study by Park et al., ${ }^{38}$ among 17 patients who underwent EDAS + bifrontal EGPS, 10 patients had excellent outcomes, 5 good outcomes, and 2 poor outcomes. The authors of this study highlighted the need for ACA territory prophylaxis and concluded that EDAS + bifrontal EGPS is an adequate surgical technique for revascularization of both the ACA and MCA territories. Patients with advancing disease can eventually suffer from frontal or occipital ischemia that is not targeted by the direct bypass procedure alone. ${ }^{25,28}$ These disadvantages must be balanced against those of the remaining options.

Various other indirect techniques have been studied in isolation. The use of omental transplantation has been studied for the alleviation of ACA and PCA circulation symptoms. In a study by Karasawa et al., ${ }^{16} 30$ children with MMD underwent omental transplantation to the ACA, PCA, or both the ACA and PCA territories. All of the 19 patients who received ACA transplants and $11(84.6 \%)$ of 13 who received PCA transplants experienced improvement in their preoperative neurological symptoms. The authors concluded that patients who demonstrate greater collateral vasculature from omental tissue actually show more rapid and complete neurological improvement, although symptoms of the PCA territory often persist. Another technique that has not been widely applied is the sole use of multiple cranial bur holes. In a study by Sainte-Rose et al. ${ }^{40} 14$ children received 10-24 bur holes in the frontotemporoparietooccipital area of each hemisphere. These authors reported complete resolution of ischemic episodes and motor improvement in those with preoperative paresis.

\section{Conclusions}

In this review of neurosurgical interventions for MMD in pediatric patients, we found that a number of techniques are effective. Although data concerning the natural history of MMD are scarce, current literature supports the treatment of symptomatic patients with MMD to reduce the risk of future stroke. In patients with typical bilateral MMD but only 1 clinically symptomatic side, perfusion studies often reveal deficits and thus bilateral treatment should be strongly considered. In patients with unilateral MMD, revascularization should be performed on the diseased side. The contralateral side should be closely monitored both clinically and angiographically, particularly if mild stenotic signs are visible given that progression of the disease is likely. ${ }^{17}$

There are a number of advantages and disadvantages to each surgical option. Direct techniques require a specific set of conditions as well as a surgeon comfortable with the technical difficulty of such microanastomosis. Based on the studies completed, the immediate revascularization induced by direct techniques reduces the risk of perioperative ischemia; however, the long-term efficacy of direct techniques in preventing ischemia has not been shown to be superior to indirect techniques. Generally, it has been shown that indirect techniques are less efficacious in elderly populations and more likely to succeed in younger patients. The explanation for such findings lies in the possibility of an age-associated reduction in angiogenic capability. In many of the aforementioned studies, aggressive postoperative radiographic studies have been pursued to either predict or verify the postoperative clinical outcome; however, the degree of revascularization has not been shown to definitively correlate with clinical outcome. Additionally, it is difficult to compare radiological results across multiple institutions given that timeframes for postoperative angiograms are not uniform. Thus, although it is still difficult to infer which procedure should be applied to specific populations of patients, the risks associated with each procedure are more clearly understood.

For the pediatric population, the debate continues regarding whether direct or indirect revascularization techniques should be preferred. To definitively assess the efficacy of each surgical technique, we need additional controlled prospective trials in which direct versus indirect revascularization techniques are compared. Molecular biology and genetic studies of MMD will lead to a greater understanding of the pathophysiology underlying MMD and perhaps enable early intervention before the development of devastating ischemia.

\section{Acknowledgments}

We convey our appreciation to the Stanford Medical Scholars Research Committee and the American Association of Neurological Surgeons Medical Student Summer Research Fellowship. Special thanks are due to Paula M. Borges and Gaurav Gupta for their help in preparing this manuscript.

\section{References}

1. Bruno A, Adams HP Jr, Biller J, Rezai K, Cornell S, Aschenbrener CA: Cerebral infarction due to moyamoya disease in young adults. Stroke 19:826-833, 1988

2. Chiu D, Shedden P, Bratina P, Grotta JC: Clinical features of moyamoya disease in the United States. Stroke 29:1347-1351, 1998

3. Fleetwood I, Steinberg GK: Moyamoya disease. Can J Neurol Sci 27:325-327, 2000

4. Golby AJ, Marks MP, Thompson RC, Steinberg GK: Direct and combined revascularization in pediatric moyamoya disease. Neurosurgery 45:50-60, 1999

5. Han DH, Kwon OK, Byun BJ, Choi BY, Choi CW, Choi JU, et al: A co-operative study: clinical characteristics of 334 Korean patients with moyamoya disease treated at neurosurgical institutes (1976-1994). The Korean Society for Cerebrovascular Disease. Acta Neurochir (Wien) 142:1263-1274, 2000

6. Hoffman HJ: Moyamoya disease and syndrome. Clin Neurol Neurosurg 99 (2 Suppl): S39-S44, 1997

7. Houkin K, Ishikawa T, Yoshimoto T, Abe H: Direct and indirect revascularization for moyamoya disease surgical techniques and peri-operative complications. Clin Neurol Neurosurg 99 (2 Suppl):S142-S145, 1997

8. Houkin K, Kuroda S, Ishikawa T, Abe H: Neovascularization (angiogenesis) after revascularization in moyamoya disease. Which technique is most useful for moyamoya disease? Acta Neurochir (Wien) 142:269-276, 2000

9. Irikura K, Miyasaka Y, Kurata A, Tanaka R, Yamada M, Kan S, et al: The effect of encephalo-myo-synangiosis on abnormal collateral vessels in childhood moyamoya disease. Neurol Res 22: 341-346, 2000

10. Ishii R, Koike T, Takeuchi S, Ohsugi S, Tanaka R, Konno K: Anastomosis of the superficial temporal artery to the distal anterior cerebral artery with interposed cephalic vein graft. Case report. J Neurosurg 58:425-429, 1983

11. Ishikawa T, Houkin K, Kamiyama H, Abe H: Effects of surgical revascularization on outcome of patients with pediatric moyamoya disease. Stroke 28:1170-1173, 1997 
12. Isono M, Ishii K, Kamida T, Inoue R, Fujiki M, Kobayashi H: Long-term outcomes of pediatric moyamoya disease treated by encephalo-duro-arterio-synangiosis. Pediatr Neurosurg 36:14-21, 2002

13. Iwama T, Hashimoto N, Tsukahara T, Goto Y, Takagi Y, Miyake $\mathrm{H}$ : Direct anastomotic bypass surgery for juvenile Moyamoya disease. Nervous System in Children 20:253-258, 1995

14. Iwama T, Hashimoto N, Tsukahara T, Miyake H: Superficial temporal artery to anterior cerebral artery direct anastomosis in patients with moyamoya disease. Clin Neurol Neurosurg 99 Suppl 2: S134-S136, 1997

15. Karasawa J, Kikuchi H, Furuse S, Sakaki T, Yoshida Y: A surgical treatment of "moyamoya" disease "encephalo-myo synangiosis." Neurol Med Chir 17:29-37, 1977

16. Karasawa J, Touho H, Ohnishi H, Miyamoto S, Kikuchi H: Cerebral revascularization using omental transplantation for childhood moyamoya disease. J Neurosurg 79:192-196, 1993

17. Kelly ME, Bell-Stephens TE, Marks MP, Do HM, Steinberg GK: Progression of unilateral moyamoya disease: A clinical series. Cerebrovasc Dis 22:109-115, 2006

18. Khan N, Schuknecht B, Boltshauser E, Capone A, Buck A, Imhof HG, et al: Moyamoya disease and Moyamoya syndrome: experience in Europe; choice of revascularisation procedures. Acta Neurochir (Wien) 145:1061-1071, 2003

19. Kikuchi H, Karasawa J: [STA-cortical MCA anastomosis for cerebrovascular occlusive disease.] No Shinkei Geka 1:15-19, 1973 (Jpn)

20. Kim CY, Wang KC, Kim SK, Chung YN, Kim HS, Cho BK: Encephaloduroarteriosynangiosis with bifrontal encephalogaleo(periosteal)synangiosis in the pediatric moyamoya disease: the surgical technique and its outcomes. Childs Nerv Syst 19:316-324, 2003

21. Kim DS, Kang SG, Yoo DS, Huh PW, Cho KS, Park CK: Surgical results in pediatric moyamoya disease: angiographic revascularization and the clinical results. Clin Neurol Neurosurg 109: 125-131, 2007

22. Kim SK, Seol HJ, Cho BK, Hwang YS, Lee DS, Wang KC: Moyamoya disease among young patients: its aggressive clinical course and the role of active surgical treatment. Neurosurgery 54:840-846, 2004

23. Kim SK, Wang KC, Kim IO, Lee DS, Cho BK: Combined encephaloduroarteriosynangiosis and bifrontal encephalogaleo (periosteal)synangiosis in pediatric moyamoya disease. Neurosurgery 50:88-96, 2002

24. Kinugasa K, Mandai S, Kamata I, Sugiu K, Ohmoto T: Surgical treatment of moyamoya disease: operative technique for encephalo-duro-arterio-myo-synangiosis, its follow-up, clinical results, and angiograms. Neurosurgery 32:527-531, 1993

25. Kinugasa K, Mandai S, Tokunaga K, Kamata I, Sugiu K, Handa A, et al: Ribbon enchephalo-duro-arterio-myo-synangiosis for moyamoya disease. Surg Neurol 41:455-461, 1994

26. Kobayashi E, Saeki N, Oishi H, Hirai S, Yamaura A: Long-term natural history of hemorrhagic moyamoya disease in 42 patients. J Neurosurg 93:976-980, 2000

27. Kono S, Oka K, Sueishi K, Sonobe M: Histopathological studies on spontaneous vault moyamoya and revascularized collaterals formed by encephalomyosynangiosis. Clin Neurol Neurosurg 99 (2 Suppl):S209-S212, 1997

28. Kuroda S, Houkin K, Nunomura M, Abe H: Frontal lobe infarction due to hemodynamic change after surgical revascularization in moyamoya disease-two case reports. Neurol Med Chir 40:315-320, 2000

29. Lim M, Cheshier S, Steinberg GK: New vessel formation in the central nervous system during tumor growth, vascular malformations, and Moyamoya. Curr Neurovasc Res 3:237-245, 2006

30. Lougheed WM, Marshall BM, Hunter M, Michel ER, SandwithSmyth H: Common carotid to intracranial internal carotid bypass venous graft. Technical note. J Neurosurg 34:114-118, 1971
31. Matsushima T, Inoue T, Katsuta T, Natori Y, Suzuki S, Ikezaki K, et al: An indirect revascularization method in the surgical treatment of moyamoya disease-various kinds of indirect procedures and a multiple combined indirect procedure. Neurol Med Chir 38 (Suppl):297-302, 1998

32. Matsushima T, Inoue T, Suzuki SO, Fujii K, Fukui M, Hasuo K: Surgical treatment of moyamoya disease in pediatric patientscomparison between the results of indirect and direct revascularization procedures. Neurosurgery 31:401-405, 1992

33. Matsushima T, Inoue TK, Suzuki SO, Inoue T, Ikezaki K, Fukui $\mathrm{M}$, et al: Surgical techniques and the results of a fronto-temporoparietal combined indirect bypass procedure for children with moyamoya disease: a comparison with the results of encephaloduro-arterio-synangiosis alone. Clin Neurol Neurosurg 99 (2 Suppl):S123-S127, 1997

34. Matsushima Y, Fukai N, Tanaka K, Tsuruoka S, Inaba Y, Aoyagi $\mathrm{M}$, et al: A new surgical treatment of moyamoya disease in children: a preliminary report. Surg Neurol 15:313-320, 1981

35. Miyamoto S, Akiyama Y, Nagata I, Karasawa J, Nozaki K, Hashimoto N, et al: Long-term outcome after STA-MCA anastomosis for moyamoya disease. Neurosurg Focus 5(5):E5, 1998

36. Mizoi K, Kayama T, Yoshimoto T, Nagamine Y: Indirect revascularization for moyamoya disease: is there a beneficial effect for adult patients? Surg Neurol 45:541-549, 1996

37. Olds MV, Griebel RW, Hoffman HJ, Craven M, Chuang S, Schutz H: The surgical treatment of childhood moyamoya disease. J Neurosurg 66:675-680, 1987

38. Park JH, Yang SY, Chung YN, Kim JE, Kim SK, Han DH, et al: Modified encephaloduroarteriosynangiosis with bifrontal encephalogaleoperiosteal synangiosis for the treatment of pediatric moyamoya disease. Technical note. J Neurosurg 106 (3 Suppl): 237-242, 2007

39. Reis CV, Safavi-Abbasi S, Zabramski JM, Gusmão SN, Spetzler RF, Preul MC: The history of neurosurgical procedures for moyamoya disease. Neurosurg Focus 20:E7, 2006

40. Sainte-Rose C, Oliveira R, Puget S, Beni-Adani L, Boddaert N, Thorne J, et al: Multiple bur hole surgery for the treatment of moyamoya disease in children. J Neurosurg 105 (6 Suppl): 437-443, 2006

41. Sakamoto H, Kitano S, Yasui T, Komiyama M, Nishikawa M, Iwai Y, et al: Direct extracranial-intracranial bypass for children with moyamoya disease. Clin Neurol Neurosurg 99 (2 Suppl): S128-S133, 1997

42. Sakamoto S, Kiura Y, Yamasaki F, Shibukawa M, Ohba S, Shrestha $\mathrm{P}$, et al: Expression of vascular endothelial growth factor in dura mater of patients with moyamoya disease. Neurosurg Rev 31:77-81, 2007

43. Scott RM: Moyamoya syndrome: a surgically treatable cause of stroke in the pediatric patient. Clin Neurosurg 47:378-384, 2000

44. Scott RM: Surgery for moyamoya syndrome? Yes. Arch Neurol 58:128-129, 2001

45. Scott RM, Smith JL, Robertson RL, Madsen JR, Soriano SG, Rockoff MA: Long-term outcome in children with moyamoya syndrome after cranial revascularization by pial synangiosis. J Neurosurg 100 (2 Suppl Pediatrics):142-149, 2004

46. Soriano SG, Sethna NF, Scott RM: Anesthetic management of children with moyamoya syndrome. Anesth Analg 77: 1066-1070, 1993

47. Stoodley MA, Steinberg GK: Omental transplantation for moyamoya disease, in Ikezaki K, Loftus CM (eds): Moyamoya Disease. Rolling Meadows, IL: American Association of Neurological Surgeons, 2001, pp 185-197

48. Suzuki J, Takaku A: Cerebrovascular "moyamoya" disease. Disease showing abnormal net-like vessels in base of brain. Arch Neurol 20:288-299, 1969

49. Suzuki Y, Negoro M, Shibuya M, Yoshida J, Negoro T, Watanabe K: Surgical treatment for pediatric moyamoya disease: use of the superficial temporal artery for both areas supplied by the anterior and middle cerebral arteries. Neurosurgery 40:324-330, 1997 


\section{Neurosurgical interventions for moyamoya disease in children}

50. Takeuchi K, Shimuzu K: Hypogenesis of bilateral internal carotid arteries. No To Shinkei 9:37-43, 1957

51. Takeuchi S, Tsuchida T, Kobayashi K, Fukuda M, Ishii R, Tanaka $\mathrm{R}$, et al: Treatment of moyamoya disease by temporal muscle graft 'encephalo-myo-synangiosis'. Childs Brain 10:1-15, 1983

52. Tripathi P, Tripathi V, Naik RJ, Patel JM: Moya Moya cases treated with encephaloduroarteriosynangiosis. Indian Pediatr 44:123-127, 2007

53. Tu YK, Liu HM, Kuo MF, Wang PJ, Hung CC: Combined encephalo-arterio-synangiosis and encephalo-myo-synangiosis in the treatment of moyamoya disease. Clin Neurol Neurosurg 99 (2 Suppl):S118-S122, 1997

54. Uchino K, Johnston SC, Becker KJ, Tirschwell DL: Moyamoya disease in Washington State and California. Neurology 65: 956-958, 2005

55. Wang MY, Steinberg GK: Rapid and near-complete resolution of moyamoya vessels in a patient with moyamoya disease treated with superficial temporal artery-middle cerebral artery bypass. Pediatr Neurosurg 24:145-150, 1996

56. Yaşargil M: Experimental small vessel surgery in the dog including patching and grafting of cerebral vessels and formation of functional and extra-intracranial shunts, in Donaghy RMP, Yaşargil MG (eds): Microvascular Surgery. St. Louis: CV Mosby Co, 1967, pp 87-126

57. Yilmaz EY, Pritz MB, Bruno A, Lopez-Yunez A, Biller J: Moy- amoya: Indiana University Medical Center experience. Arch Neurol 58:1274-1278, 2001

58. Yonekawa Y, Kawano T: Follow-up study of 632 cases in spontaneous occlusion of the circle of Willis registered from 1983 to 1991, in Yonekawa Y (ed): The Research Committee on Spontaneous Occlusion of the Circle of Willis (Moyamoya Disease) of the Ministry of Health and Welfare, Japan: Annual Report 1991. Osaka, Japan: National Cardiovascular Center, 1992, pp $41-47$

59. Yoshioka N, Tominaga S: Cerebral revascularization using muscle free flap for ischemic cerebrovascular disease in adult patients. Neurol Med Chir 38:464-468, 1998

60. Zipfel GJ, Fox DJ Jr, Rivet DJ: Moyamoya disease in adults: the role of cerebral revascularization. Skull Base 15:27-41, 2005

Manuscript submitted November 16, 2007.

Accepted December 4, 2007.

This work was supported in part by funding from the William Randolph Hearst Foundation, Bernard and Ronni Lacroute, and Russell and Elizabeth Siegelman.

Address correspondence to: Gary K. Steinberg, M.D., Ph.D., 300 Pasteur Drive R281, Stanford, California 94305-5327. email: gsteinberg@stanford.edu. 